\title{
HIGH-RESOLUTION WAVEFRONT CONTROL USING MICROMIRROR ARRAYS
}

\author{
Julie A. Perreault and Thomas G. Bifano
}

Boston University, College of Engineering, Boston MA, 02215

\begin{abstract}
Silicon deformable mirrors have the potential to modulate spatial and temporal features of an optical wavefront. This capability is critical for systems in which aberrations affect optical beam quality, including many applications in laser communication, medical imaging, astronomy, and target tracking. The main goal of the work presented here was to design, build, and test a 1024 actuator wavefront controller for real-time modulation of an optical wavefront.
\end{abstract}

\section{INTRODUCTION}

This work builds on the heritage of several generations of silicon micro-machined deformable mirrors and spatial light modulators that were fabricated over the past several years. Previous work focused on smaller SLM arrays. Results from these devices laid the foundation for a $32 \times 32$ array device with regard to design ${ }^{1}$ manufacturing ${ }^{2},{ }^{3}$ and characterization ${ }^{4}$. New work includes layout, fabrication, and packaging of this kilo-pixel device, characterization of high-speed mirror dynamics in air and in vacuum, and integration of the device in an optical test bed for high-speed holographic compensation of aberrations.

\section{DEFORMABLE MIRROR}

The kilo-pixel SLM is made up of 1024 individually addressable surface-normal electrostatic actuators with center posts that support individual optical mirror segments. Each electrostatic actuator consists of a silicon membrane anchored to the substrate on two sides above a silicon electrode (Figure 1).

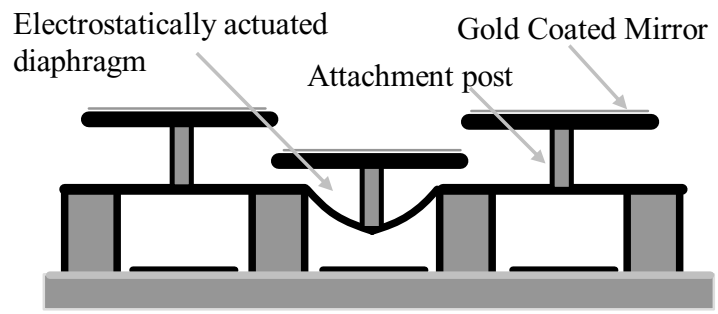

Figure 1. Schematic cross section of a gold coated spatial light modulator with a central deflected actuator.

A post centered on each actuator supports a $338 \mu \mathrm{m}$ x $338 \mu \mathrm{m}$ $\mathrm{x} 3 \mu \mathrm{m}$ optically coated mirror segment. The SLM has an aperture of $10 \mathrm{~mm}$, actuator stroke of $2 \mu \mathrm{m}$, and a position repeatability of $3 \mathrm{~nm}$. Devices were fabricated with a combination of silicon surface micromachining and several custom fabrication processes to ensure optical quality. Chemo-mechanical polishing was used to remove surface roughness and ion machining was used to remove curvature. A stress-free gold coating process was also developed to increase reflectivity. The devices were packaged by wire-bonding 1024 bond pads around the periphery of the device to a custommade ceramic package (Figure 2).

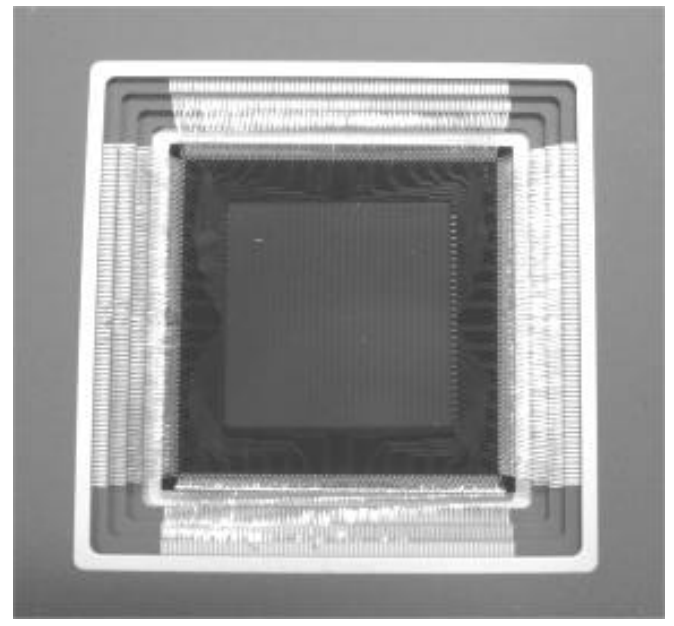

Figure 2. Photograph of kilo-pixel SLM

\section{BACKGROUND}

There are currently a number of research groups working on the development of MEMS-based deformable mirrors for adaptive optic correction. Early work in this field included aluminum spatial light modulators demonstrated by Texas Instruments more than twenty years ago ${ }^{5}$. These one-bit, segmented, phase-adjusting devices predated the more well-known amplitude modulating micromirror arrays that were developed by Texas Instruments for projection display applications. More recently, segmented micromirror devices based on silicon micromachining were pioneered by the Air Force Research Laboratory, using the SUMMiT IV process. This group was among the first to report the use of micromirrors for adaptive optics compensation ${ }^{6}$.

Only a few efforts to produce MEMS deformable mirrors have resulted in devices with sufficient stroke, resolution, and optical quality (smoothness, flatness, and reflectivity) for use in optical imaging applications. One of these, the first commercially available MEMS-based deformable mirror was developed at Delft University and later commercialized ${ }^{7}$. This device was comprised of a silicon nitride membrane suspended over an array of addressable electrodes ${ }^{8}$. Subsequently, similar MEMS-based deformable mirrors were designed and manufactured at Stanford University ${ }^{9}$, and later commercialized ${ }^{10}$. A two-level silicon surface micromachining approach was employed by researchers at Boston University to produce MEMS deformable mirrors using an original architecture described in Figure $1^{11}$. These devices were manufactured at a commercial MEMS foundry ${ }^{12}$ and were later commercialized $^{13}$.

Recently, many researchers have reported on the use of these and other MEMS-based deformable mirrors in adaptive optics applications. Mirrors of different architectures and designs have been compared in recent reviews to delineate their optical and electromechanical performance ${ }^{14,15}$. 


\section{DEVICE CHARACTERIZATION}

Mirror characterization included measurements of electromechanical actuator performance and optical surface contour. Dynamic measurements included frequency response in air and vacuum, motion repeatability, and strobed surface mapping of a single spatial light modulator segment.

For closed loop operation, it is important to know the temporal response of the micro mirror system. The bandwidth of a spatial light modulator (SLM) was measured in air using a laser Doppler vibrometer (LDV). An individual mirror segment was driven with a sinusoidal $1 \mathrm{Vpp}$ signal offset by $100 \mathrm{~V}$ while monitoring velocity with the LDV. Velocity measurements were made over a discrete range of input frequencies and integrated to yield displacement data.

Frequency response measurements made with a high speed Doppler laser vibrometer indicate an actuator bandwidth of approximately $60 \mathrm{kHz}$ at low pressure. There was considerable air damping when operated at atmospheric pressure (Figure 3).

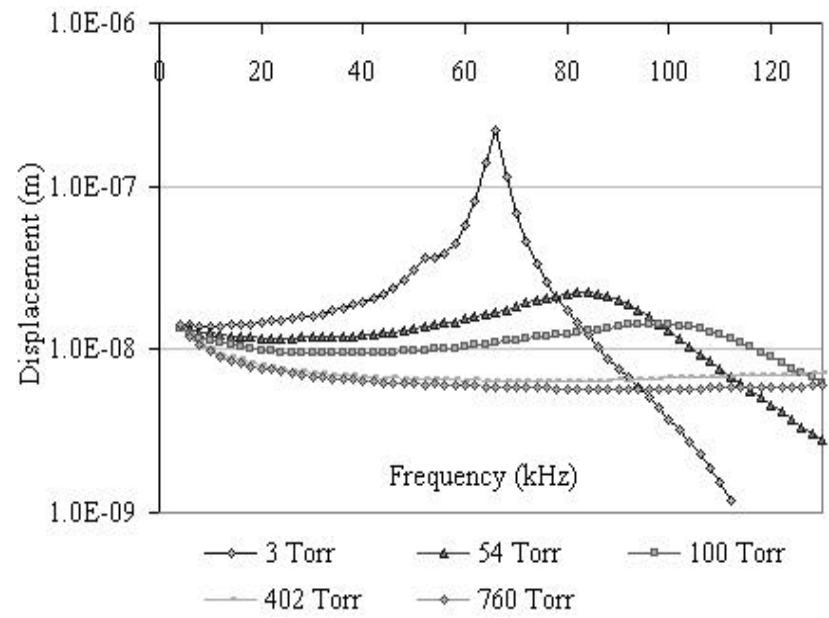

Figure 3. Mirror response characterization in air and in partial vacuum, illustrating the $60 \mathrm{kHz}$ natural frequency and the effects of air damping.

An important measure of device performance is position repeatability, defined as the standard deviation of a set of measurements of deflection made on a single actuator subjected to repeated, identical voltage excursions. Position repeatability was measured for a single actuator on a continuous membrane mirror through ensemble averaging of the data from a series of tests.

Position repeatability measurements were made with a dynamic single point interferometer that uses a focused laser beam to measure normal displacement with a position resolution of $2.5 \mathrm{~nm}$, a range of $\pm 250 \mu \mathrm{m}$, a frequency bandwidth of $0-133 \mathrm{kHz}$, and a lateral averaging area of $\sim 30 \mu \mathrm{m}$. Measurements were made over several hours while the mirror was enclosed in a chamber at a partial pressure of 200Torr. Repeatability was demonstrated to be within $4 \mathrm{~nm}$. (Figure 4), roughly corresponding to the noise floor of the measurement system.

A similar repeatability measurement was made at the Jet Propulsion Laboratory (JPL) using an instrument with higher measurement resolution. The JPL laser metrology measurement system was used to measure the small signal actuator response with an offset of $10 \mathrm{~V}$. Response data was recorded for sinusoidal input excitation frequency of $35 \mathrm{~Hz}$ and amplitudes ranging from $1 \mathrm{Vpp}$ to $2 \mathrm{Vpp}$ in $0.1 \mathrm{Vpp}$ steps. It was found that the actuator position repeatability was approximately $13 \mathrm{pm}$ (one standard deviation), over that measurement range (corresponding to excursions ranging from $\sim 2.5 \mathrm{~nm}$ at $1 \mathrm{Vpp}$ to $\sim 5.0 \mathrm{~nm}$ at $2 \mathrm{Vpp}$ ). This repeatability measurement corresponded to the approximate noise floor of the instrument at this frequency.

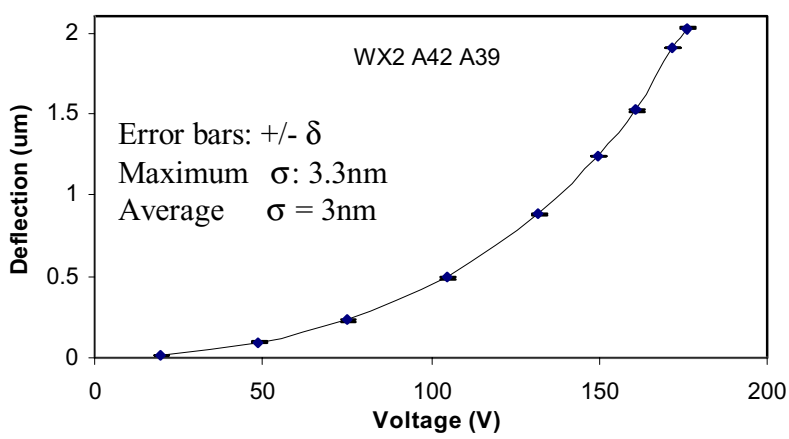

Figure 4. Voltage versus deflection measurements of a single actuator on a spatial light modulator with a repeatability of $\sim 4 \mathrm{~nm}$.

To study high-speed system response, a strobed interferomeric surface mapping instrument was used to characterize the complex motion of a mirror segment in real time (Umech Technologies MMA-G2). A custom vacuum chamber was designed and built to fit in the measurement system. A $100 \mathrm{~V}$ square wave input was applied to a single pixel on the SLM and the response was measured while the mirror was maintained at 38Torr or 349Torr. Strobed interferometric contour mapping measurements showed considerable flexure of the mirror segment in response to step changes in actuator position at lower pressures (Figure 5).

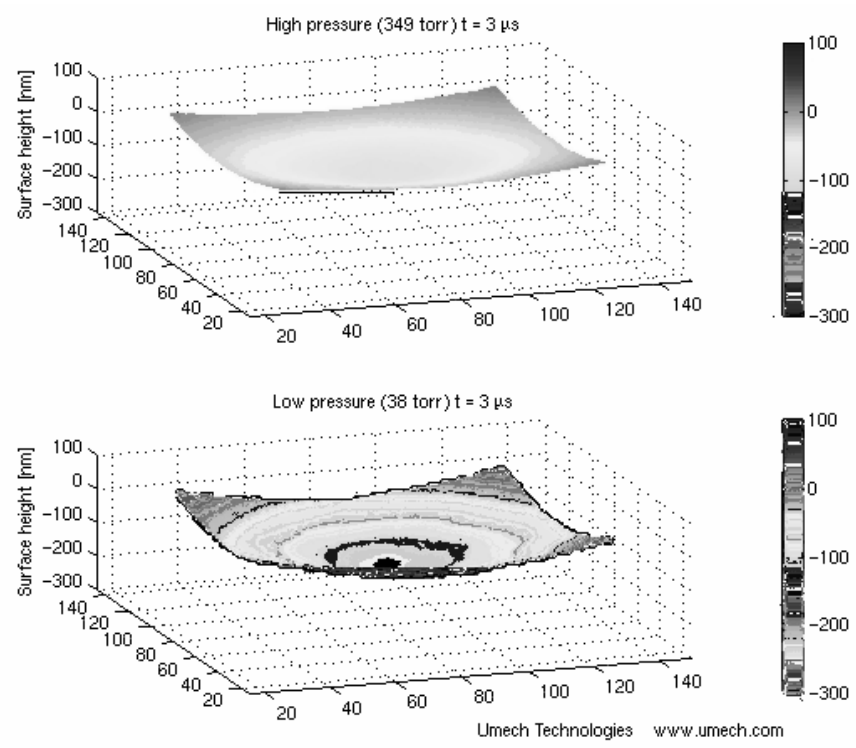

Figure 5. Still frame capture from a dynamic measurement of mirror pixel step response at 349Torr (top) and at 38Torr (bottom) recorded 3 ?s after a step change on the actuator input.

\section{WAVEFRONT CONTROL}

After characterization was completed, the SLM was integrated into a laboratory test bed and a field test bed for laser communication at Lawrence Livermore National Laboratories (LLNL). 
The testbed was developed for use with this kilo-pixel MEMS $\mathrm{SLM}^{16}$. A holographic phase conjugation engine made use of the SLM to dramatically reduce the effects of horizontal path aberrations at a frame rate of $\sim 890 \mathrm{~Hz}$. A diagram of the optical test bed is shown in (Figure 6). The system is comprised of a SLM, CCD, Laser, specialty phase-plates, quarter and half wave plates (QWP, HWP), hologram splitter, and bench top optics.

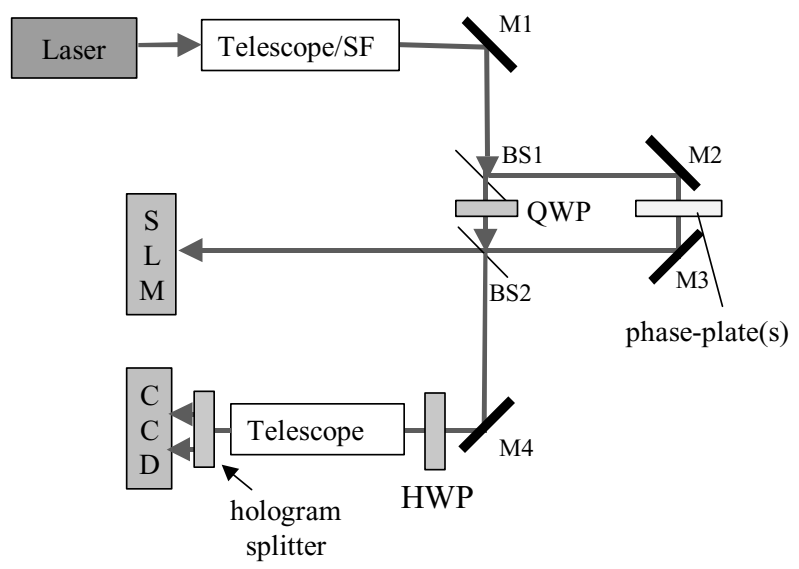

Figure 6. Optical Test bed for open and closed loop aberration correction for laser communication.

A 50/50 beam splitter (BS) is used to create a reference beam and probe beam from a vertically polarized laser. Specialty phase plates, designed to simulate atmospheric turbulence with a Kolmogorov phase structure function, were used to introduce aberration into the probe beam path. The reference beam is passed through a QWP to change it's vertical polarization to circular, which is used to form to interferograms with a $\pi / 2$ phase shift. The aberrated probe beam is reflected from the SLM and recombined with the circularly polarized reference beam.

The recombined beams are then passed through a half wave plate to rotate the polarization by $\pi / 4$. This rotation does not affect the phase shift between the horizontal and vertical components of the reference beam. It does produce equal amplitude horizontal and vertical components in the reference beam. A holographic splitter is used to spatially separate the pair of interferograms, which are then imaged onto the CCD camera with a telescope. This type of design was implemented to perform adaptive correction that exploits parallel control of all SLM pixels using direct optical feedback.

Strehl ratio, the peak intensity of an aberrated point image normalized by the peak intensity of an unaberrated point image, was measured in both the lab test bed and in the field, in work that is not yet published. The Strehl improvement in the lab was approximately a factor 25 which is an absolute Strehl of approximately $60 \%$. Improvements in the field had an improvement by roughly a factor of 8 , or an absolute Strehl of approximately $46 \%$, which is still considered very good. The artificial distortions created on the lab bench setup by (rotating) phase screens were significantly more severe than the real atmospheric distortions in the field. This accounts for the higher improvement factor in the laboratory.

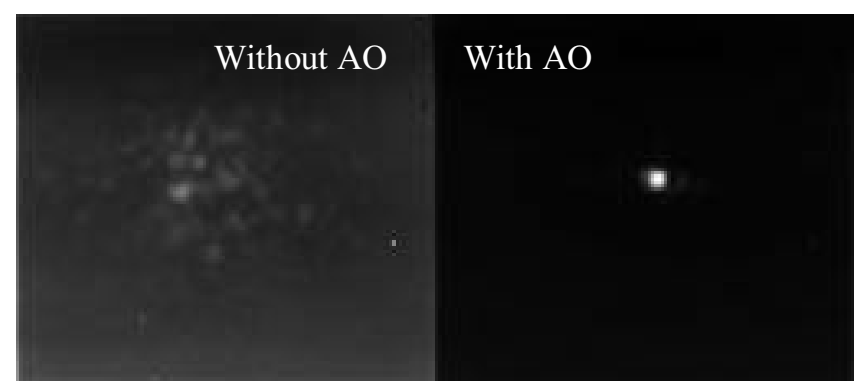

Figure 7. Far-field image formed using a laser communication system with the kilo-pixel SLM, running open loop at a frame rate of $890 \mathrm{~Hz}$. Field tests were conducted at LLNL with support from DARPA via the Coherent Communication, Imaging, and Targeting Program (CCIT). Figures provided courtesy of Lawrence Livermore National Laboratories (LLNL).

\section{CONCLUSIONS}

Static and dynamic behavior of individual actuators in the device have been measured, and their characteristic stroke of 2 ? $\mathrm{m}$, repeatability of $\sim 13 \mathrm{pm}$, and frequency response $>>1 \mathrm{kHz}$ have proven suitable for wavefront correction in an aberrated optical system. These high-resolution wavefront correctors may become an important element in future adaptive optical compensation systems.

\section{ACKNOWLEDGMENTS}

Travel support has been generously provided by the Transducers Research Foundation and by the DARPA MEMS and DARPA BioFlips programs. Support for the development and testing of SLMS was provided by a research contract from DARPA's Coherent Communications, Imaging and Targeting Program, and that work was conducted in collaboration with Boston Micromachines Corporation. Repeatability testing at JPL was supported by a grant from NASA through the Terrestrial Planet Finder Program. Control experiments were supported by an Army Research Office contract for Communicating Networked Control Systems, through a Multi-University Research Initiative. Graduate fellowship support was provided in part by the Army Research Laboratory, through the Boston University Photonics Center Co-op Program.

\footnotetext{
${ }^{1}$ Mali, R. K., Bifano, T. and Koester, D. A., "Design-based approach to planarization in multilayer surface micromachining," $\mathbf{J}$. Micromech. Microeng. [9], pp. 294-299, 1999

2 Horenstein, M.N., Bifano, T.G., Mali, R. K., Vandelli, N., "Electrostatic Effects in Micromachined Actuators for Adaptive Optics," Journal of Electrostatics [42], pp. 69-82, 1997

${ }^{3}$ Bifano, T. G., Johnson,, H. T, Bierden, P. and Mali, R. K., "Elimination of Stress-Induced Curvature in Thin-Film Structures" J. Microelectromechanical Systems, [11], pp 592-597, 2002

${ }^{4}$ Bifano, T. G., Perreault, J., Mali, R. K., and Horenstein, M. N., "Microelectromechanical Deformable Mirrors," Journal of Selected Topics in Quantum Electronics, [5], pp. 83-90, 1999

${ }^{5}$ Hornbeck, L. J., “128 x 128 deformable mirror device," IEEE ED [30], pp. 539-545, 1983
} 
${ }^{6}$ Roggemann, M. C., Bright, V. M., Welsh, B. M., Hick, S. R., Roberts, P. C., Cowan, W. D., and Comtois, J. H., "Use of microelectro-mechanical mirrors to control aberrations in optical systems: theoretical and experimental results", Optical Engineering, [36], pp.1326-1338, 1997

${ }^{7}$ Flexible Optical B V, Delft, Netherlands Technologies

${ }^{8}$ Vdovin, G and Sarro P. M., "Flexible mirror micromachined in silicon,” Applied Optics [34], pp. 2968-2972, 1995

${ }^{9}$ Mansell, J. D., and Byer, R. L., "Micromachined silicon deformable mirror," Proc. SPIE Vol. 3353, Adaptive Optical System Technologies; Domenico Bonaccini, Robert K. Tyson; Eds. pp. 896-901, 1998

${ }^{10}$ Intellite Corporation, Albuquerque, NM

${ }^{11}$ Krishnamoorthy, R., Bifano, T. G., Vandelli, N., and Horenstein, M., "Development of MEMS deformable mirrors for phase modulation of light," Optical Engineering [36], pp. 542-548, 1997

${ }^{12}$ Currently MEMSCAP, Inc. Durham, NC

${ }^{13}$ Boston Micromachines Corporation, Watertown, MA

${ }^{14}$ Krulevitch. P., Bierden, P. A., Bifano, T., Carr, E., Dimas, C., Dyson, H., Helmbrecht, M., Kurczynski, P., Muller, R., Olivier, S., Peter, Y., Sadoulet, B., Solgaard, O., and Yang, E. H., "MOEMS spatial light modulator development at the Center for Adaptive Optics," Proc. SPIE Vol. 4983, MOEMS and Miniaturized Systems III, James H. Smith, Peter A. Krulevitch, Hubert K. Lakner, Eds., pp. 227-234, 2003.

${ }^{15}$ Weyrauch T., Vorontsov M. A., Bifano T. G., Hammer J. A., Cohen M., and Cauwenberghs G., "Microscale adaptive optics: wavefront control with a $\mu$-mirror array and a VLSI stochastic gradient descent controller," Applied Optics, [40] 24 pp. 42434253, 2001

${ }^{16}$ Baker, K. L., Stappaerts, E. A., Wilks, S. C., Young, P. E., Gavel, D. T., Tucker, J. W., Silva, D. A. and Olivier, S. S., "Openand closed-loop aberration correction by use of a quadrature interferometric wavefront sensor", Optics Letters, [29], pp. 47-49, 2004 